\title{
Experiences with the implementation of measures and tools for road safety improvement
}

\author{
Miroslava Mikusova \\ Professor Assistant, Department of Road and Urban Transport, Faculty of Operation and \\ Economics of Transport and Communications, University of Zilina, Slovakia
}

\section{Jozef Gnap}

Professor, Department of Road and Urban Transport, Faculty of Operation and Economics of Transport and Communications, University of Zilina, Slovakia

\section{SUMMARY}

The paper presents an overview on the road safety measures implemented in the framework of the "SOL - Save our lives" project. It contains summarization of general knowledge regarding the efficiency of the measures applied and conclusions from the analyses of developed strategies and action plans, including common issues, strengths and weaknesses of developed tools and puts these in the context of wider European Road Safety strategies.

The purpose of the paper is to provide recommendations for an effective professional development of road safety programs at community level in the context of sustainable mobility.

\section{INRODUCTION}

In 2010, the European Union renewed its commitment to improving road safety by setting a target of reducing road deaths by $50 \%$ by 2020, compared to 2010 levels (Adminaite et. al, 2015). This goal was supported also by the SOL „Save our Lives - A Comprehensive Road Safety Strategy for Central Europe“" project.

Main goal of the project was jointly develop a strategy of road safety to support the Central European regions in catching up with highest EU standards in road safety (Bliss, 2009):

- Assess the problem, policies and institutional settings relating to road safety and the capacity for road injury prevention.

- Strengthen institutions and create effective horizontal and vertical multi-sector partnerships.

- Prepare regional/local strategies and action plans and allocate endogenous resources to address the problem.

- Implement specific actions to prevent road traffic crashes, minimize injuries and their consequences.

- Create a greater level of awareness, commitment and informed decision-making at all levels.

- Develop replicable tools for central European space and the EU. 
- Put road safety policies in the context of promoting sustainable forms of mobility.

SOL was linked to global work - it assisted communities in Central European space implementing the main recommendations of the World report on road crash injury prevention (World Bank), including an overall increase of political commitment towards road safety, developing activities based on evidence rather than "ad-hoc", developing strategies and action plans, allocating resources to the main road safety risks, implementing local projects, monitoring and evaluating impacts.

The aim of the project SOL was to prevent road crashes, deaths and injuries in the Central Europe Space. In 12 pilot areas of 7 countries (Poland, Czech Republic, Slovakia, Hungary, Slovenia, Italy and Austria) targeted strategies were developed implementing effective programmes to build a transnational road safety network. The pilot actions focused on different aspects were addressed to the target groups.

Project work was based on pyramid model shown in Fig. 1.

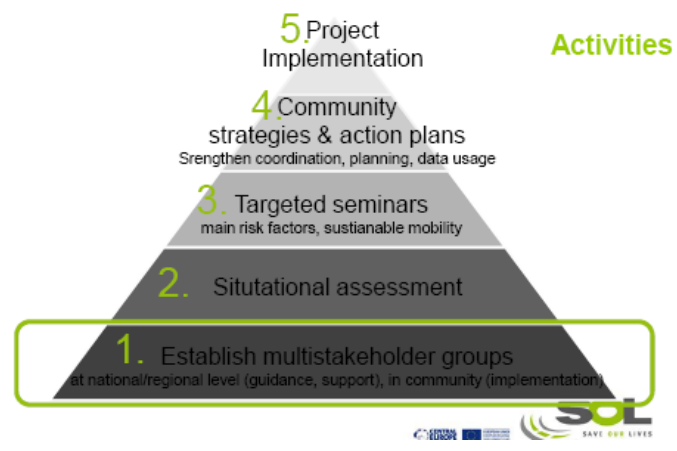

Fig. 1 - Pyramid model of the SOL project activities (SOL, 2015)

Firstly, a top-down input was applied, as the experts teams reached the local communities and recognized the most active ones in order to supply them with the necessary professional skills and tools to get the awareness of the focal issues concerning their own community.

Secondly, the local communities, once endowed with the above described skills and tools, were fostered to get started a stable connection with the upper level in order to communicate the main discovered needs (also thanks to the skills built in the top-down stage) and get an active role in building an action plan and a consequent pilot action, with a bottom-up input.

This cross of top-down and bottom-up inputs created a vertical network made of interconnected realities, in permanent cooperation, sharing useful data and knowledge.

\section{SOL ROAD SAFETY TOOLS}

Project SOL aimed to prevent road crash deaths and injuries and increase sustainable mobility in the participating SOL communities in Central Europe by supporting the development of targeted strategies and action plans, up-skilling road safety professionals, 
implementing effective road safety interventions and building a transnational road safety network.

In each of the selected areas individual community road safety strategies and action plans have been produced and pilot road safety activities established. The focus of the project was on implementing the measures in the road safety strategy and action plans and/or on transferring regional road safety programmes to the local level through communicators who fostered local awareness and action.

A monitoring system has been set-up in order to control the performance of each pilot activity. This will allow intermediate adjustments of methodology and lay the ground for a later evaluation of the tools regarding their applicability within Central European Space. Tools, approaches and strategies that could prove their effectiveness will form the basis for the SOL manual that is a key output from the project.

Within the action plans SOL financed some pilot projects as an application of the local process towards road safety improvement.

\subsection{SOL Strategies}

The SOL partner strategies were very different, which reflects the different situations in the countries. There are, however, many central principles of road safety management that are relevant across the strategies and it is these that we identify here in relation to the above strategies.

Any local strategy is very dependent upon the national situation. If there is no desire for road safety nationally, then it can be difficult to generate enthusiasm and support locally. This is the case in many of the strategies and the difficulties noted. It is often the case that road safety is not a high local priority and measures are needed to attempt to raise the priority. One way of getting local support, despite lack of national interest, is to engage with the public and apply pressure to local government through the media. In most societies road deaths and injuries are personal tragedies that are news-worthy and bereaved families can provide human interest stories that pressure local politicians to take action. Our reviews have noted that more engagement with the public might be beneficial.

Evidence shows that road safety programmes that have a high-level Champion are more likely to be successful. Some of the above strategies do have Champions, and it is the level of this commitment that will be critical to the success of the projects. Where no obvious Champion is identified, we have noted this omission and recommended that an effort is made to identify one.

The Partners cannot deliver these projects on their own and the number, quality and commitment of the stakeholders will be a critical success factor. We have often 
recommended that additional stakeholders would be beneficial. We have also recommended that stakeholder committees be set up with regular meetings to ensure good communication and to maximise the involvement of supporting parties.

One of the most important factors is ensuring that, as far as possible, the projects are based on evidence that there is a road safety problem. This required good crash data and a sound analysis. Unfortunately this is not always available, but in every case we have noted that a system is proposed that leads to improved crash data over time. We have convinced the Partners that this is necessary and hope that a more quantitative analysis and problem identification process will be developed in the future.

Managing and coordinating activities can be challenging. In few cases this exists and it is a crucial condition for strategy success. We recommend that a management committee be set up, with sub committees if appropriate (e.g. for engineering, publicity, campaigns, etc.). This needs to meet regularly and have minutes that record actions etc.

Stakeholders need to commit to the projects and an inspiring strategy can help with this. Who would not want to halve road deaths? Road Safety is often a 'background' activity; something that happens to others and not me. Some effort in making everyone aware of the size of the problem and that road death does happen to real people all the time can help the project enormously. These deaths and injuries are preventable - we know how to do it, it just needs commitment. Raising the profile is time well spent. Again the media can help in promoting the strategy and target reductions and get general public support.

Projects often fail because of a lack of momentum and commitment. Getting stakeholders to deliver to time and quality, especially in these difficult financial times, is a big challenge. The best way is to withhold payments, which requires the project manager to hold budgets. This is not always possible, so ways are needed to embarrass or otherwise pressurise stakeholders to deliver.

The projects often do not consider the legacy and in some cases we have recommended a little more consideration is given to ensuring the processes get embedded in the local setups. Leaving a legacy of continually improving road safety is probably the most important element of these projects.

\subsection{SOL Action Plans}

The Action Plans from the partners were varied, as would be expected given the different situations. There are, however, some common major issues which we feel are important in delivering a successful project.

Getting support and commitment for the project is key to success. The national situation is covered in the strategies so it is the local situation which is most significant here. The best 
Plans will have good and well managed involvement from stakeholders, which often means have a structured approach with groups, committees and minuted actions etc. A Project Management Group is a good way of maximising stakeholder commitment.

As well as managing the stakeholders, getting the right ones involved is also important. All the Action Plans have a list of several stakeholders and they are rightly different in different countries and situations. Sometimes, however, broadening the stakeholders can be beneficial. For example involving the media can be very helpful, and publicity etc is not always mentioned in the Action Plans.

The Action Plans vary from one measure to a large number of interventions. Having one or few measures allows focus and probably gives the best chance for success in the area. On the other hand if there is a wide range of interventions then the chances are that some of them will not go well while others will be successful. Having a small focused project, however, does allow for monitoring and it is often easier to capture the legacy from the work.

Getting commitment from stakeholders is always challenging and given the different stakeholders the ways to get commitment can vary. If the project has a Champion (e.g. the Mayor), this can be very helpful in getting commitment.

Road safety actions often affect a wide range and number of road users, but it is helpful to know what particular groups are likely to benefit most. This information is useful when designing the intervention, so it can be targeted and gain maximum impact. Using crash data to identify the road safety problems of different groups is a key factor in designing effective action plans.

Evidence shows that road safety programmes achieve more when they have clear and easily supported objectives. Including targets, e.g. for casualty reductions, can also be good. Action Plans work best when the activities are clear and there is a stakeholder who is clearly responsible for each activity. Having timescales and a clear idea about deliverables is also important. Clarity about the final report, what it contains and who is writing each section of it can help in delivering a successful project.

Money talks. The best way to get things done is to pay for them and hence have control of the quality and timescales. Relying on stakeholders to do things with their own resources is a lot more difficult. Finances therefore are critical and allocation of budgets (if any) needs careful attention.

\section{EVALUATION OF EFFECTIVENESS OF DEVELOPED TOOLS}

After evaluating the overall effectiveness of the tools developed within the SOL project by international team of road safety experts it is possible to conclude following: 
- One of the main and the most important results of the SOL project was the elaboration of local road safety strategies.

- The creation of a permanent Road Safety Expert Group could be considered also very important. The biggest challenge is to get the appropriate political commitment.

- The road safety campaigns alone (without associated enforcement) were not found to reduce accident numbers and therefore most likely would not prove to be cost-effective.

- In the SOL project a lot of pilots were focused on improvement of the child safety. Unfortunately, the target group was not identified appropriately in many cases; e.g. it was not obvious if that the target group was the pre-school children or the school children. What is more, the pilots in some cases consisted of a lot of different tools (infrastructure safety, campaigns, etc.) and different target groups (children, truck drivers, etc.)

- From the point of view of tools efficiency it is outstandingly important to focus clearly on a target group and to use different methods according to the characteristics of the target group.

- Some further characteristics make the evaluation of tools very complicated. The period of time was very short, in most cases only some months. It is almost impossible to hope for significant change in the field of behavior during some months. Nevertheless, the local road safety strategy elaborated in the framework of SOL makes it possible to continue the work (in this case, campaigns) beyond SOL also. In this case the evaluation could be carried out on the basis of a longer period of time; the probability of positive change in behavior is much higher than some months.

- Only the campaigns combined with enforcement were found to reduce accidents. Campaigns alone do not seem to have any effect at all.

- Only some studies have used roadside surveys in order to evaluate the effects on drinkdriving, whereas other studies have used self-reported drink-driving, which must be assumed not to be an optimal measure. Only some studies have used roadside surveys in order to evaluate the effects on drink-driving, whereas other studies have used selfreported drink-driving, which must be assumed not to be an optimal measure.

- Despite some methodological weaknesses (for example self-reported behavior, effects from campaigns not separated from effects from enforcement, etc.) it is possible to change road user behavior by means of information and campaigns. Greater changes in behavior are achieved when information campaigns are combined with increased police enforcement than when they are not. The use of television as a medium in the campaign appears to lead to greater changes in behavior than other media would do.

- Prepare regional/local strategies and action plans and allocate endogenous resources to address the problem.

\section{CONCLUSIONS}


Although progress has been made in EU countries in reducing the toll of road traffic crashes over the past decade, much remains to be done, especially the need to raise safety standards in countries with above average casualty rates to the level that pertains in the best performing countries. Some 1.25 million people die each year as a result of road traffic crashes (WHO, 2015). The development of road safety policy for the EU for the period 2011-2020 is aimed at reducing road deaths by half over this period. At the global level, 2011-2020 has been proclaimed by the United Nations as a Decade of Road Safety with a goal to stabilize and then reduce the forecast level of road traffic fatalities around the world by increasing activities conducted at the national, regional and global level.

Whilst the main focus of EU road safety policy is at national level, the implementation of policy requires action at regional and local levels. In particular, improving road safety requires the support of the whole of society, not just government and official organizations. Understanding of risk and acceptance of the need for all road users to take responsibility for safe behaviour, whilst the designers and operators of the road system are responsible for building in safety and accommodating human error, are the fundamental principles underpinning a Systems Approach to road safety. These conclusions are confirming the findings of road safety study (Breen, 2015) created by independent expert for review of the EU Road Safety Policy Orientation.

The SOL project, through a bottom-up approach, will provide the foundation stones at local level on which broader road safety policy can be built. Involvement of local communities is a critical factor in producing a safety conscious culture in society where risk is understood and action taken to reduce it. The outputs from the pilot programmes will provide lessons that are transferable to other countries and regions thus fulfilling the transnational aims of the SOL project.

\section{REFERENCES}

ADMINAITE, D., ALLSOP, R. y JOST, G. Ranking EU progress on road safety. $9^{\text {th }}$ Road Safety Performance Index Report. ETSC, 2015.

BREEN, J. Country guidelines for the Conduct of Road Safety Management Capacity. ETSC, 2015.

BLISS, T., BREE, J. Reviews and the Specification of Lead Agency Reforms, Investment Strategies and Safe System Projects. The World Bank Global Road Safety Facility, Washington DC, 2009.

(2015) The SOL project website. [Online]. Available: http://www.sol-project.eu/. 